\title{
Modeling and estimating the odometry error of a mobile robot
}

\section{Conference Paper}

Author(s):

Martinelli, Agostino

Publication date:

2001-07

Permanent link:

https://doi.org/10.3929/ethz-a-010107221

Rights / license:

In Copyright - Non-Commercial Use Permitted

Originally published in:

IFAC Proceedings Volumes 34(6), https://doi.org/10.1016/S1474-6670(17)35209-6 


\title{
MODELING AND ESTIMATING THE ODOMETRY ERROR OF A MOBILE ROBOT
}

\author{
Agostino Martinelli* \\ * Dipartimento di Informatica, Sistemi e Produzione, Università \\ degli Studi di Roma "Tor Vergata", Via di Tor Vergata, 110 - \\ 00133 Roma (Italy)
}

\begin{abstract}
:
This paper presents both an error modeling of an odometry system for a synchronous drive system and a possible procedure in order to evaluate it. The odometry error is modeled by introducing four parameters. Two parameters characterize the two non-systematic components (translational and rotational). The other two parameters characterize the translational and rotational systematic components. The non-systematic errors are expressed in terms of a covariance matrix which depends both on the previous four parameters and on the path followed by the mobile robot. In contrast to previous approaches which require to assume a particular path (straight or circular) in order to compute this covariance matrix, here general formulas are derived. In these formulas, applicable to any path, the trajectory of the robot motion appears as a function of the curve length.
\end{abstract}

Keywords: odometry, mobile robots, error modeling, error estimating

\section{INTRODUCTION}

Determining the odometry errors of a mobile robot is very important both in order to reduce them, and to know the accuracy of the state configuration estimated by using encoder data. Odometry, in fact, is the most widely used navigation method for mobile robot positioning. This method actually is inaccurate since the error in the position estimation integrates over the path. However the encoder data are extensively used in the localization process by fusing these data with data coming from another (or several) sensor. Clearly any fusion architecture needs to know the accuracy of the estimate of each sensor in order to weigh all the data in a proper manner. The most used fusion algorithm is the Extended Kalman Filter (see for example (Anousaki and Kyriakopoulos, 1999) and references therein). In particular when the fusion regards the encoder data the accuracy is completely described by the odometry error covariance matrix, $Q$.
Odometry errors can be both systematic and non-systematic. While systematic errors depend only on the mobile robot independently of the environment where the robot moves, the nonsystematic errors depend on the environment and drastically change by changing environment.

In a series of papers Borenstein and collaborators (Borenstein, 1994a; Borenstein, 1995a; Borenstein, 1995b; Borenstein, 1996; Borenstein, 1994b; Borenstein, 1998; Zhejun, 1995) investigated on the possible errors sources of both kind of errors. A review of all the types of these sources is given in (Borenstein, 1998) to which the reader is addressed.

Regarding the systematic errors in differential drive mobile robots there are two dominant error sources: unequal wheel diameters and the uncertainty about the effective wheel base. In the work by Borenstein and Feng (Borenstein, 1994b), a calibration technique called UMBmark test has 
been developed to calibrate for systematic errors of a two wheel robot. This method has been used by other authors (Chong, 1997).

Goel, Roumeliotis and Sukhatme (Goel, 1999) used another calibration procedure to compensate systematic errors. They referred to the differential drive mobile robot Pioneer AT. They measured (when the robot was sitting on a box and the wheels rotated freely in the air) the actual velocities of the wheels and the velocity measurements from the encoders. In this way they found a relationship between the velocity returned by encoders and the actual velocity (measured by using a precise tachometer). Moreover they measured the effective axle length due to skid steering which differs from that given by the specifications for the robot.

Finally Roy and Thrun (Roy, 1999) suggested an algorithm that uses the robot's sensors to automatically calibrate the robot as it operates.

In a series of papers Borenstein ((Borenstein, 1998) and reference therein) suggested also a method to improve the accuracy of the odometry data by reducing the effect of the non-systematic errors. With this method, called IPEC (Internal Position Error Correction), it was possible to detect and correct odometry errors without inertial or external-reference sensors. In particular he implemented the IPEC method on the special designed mobile robot platforms MDOF (Multi Degree of Freedom) (Borenstein, 1995a) and Omnimate (Borenstein, 1998). Experimental results showed that the accuracy achieved with the IPEC method was one to two orders of magnitude better than that one of systems based on conventional dead-reckoning.

Wang (Wang, 1988) analyzed the non-systematic errors from a theoretical point of view and computed the odometry covariance matrix $Q$. He referred to a differential drive mobile robot. In order to evaluate this matrix he divided the entire path in $N$ small elementary paths. To compute the covariance matrix he had to make some assumptions about the type of the elementary path. In particular he assumed a circular path. Moreover, since the updated robot position depended non-linearly on the changes in the translation and orientation (measured by the encoders), he had to introduce another approximation. He called the non-linear term appearing in the updated position as the adjustment factor. He analyzed three different cases depending on the considered approximation for this factor. In particular he considered a Taylor approximation of the adjustment factor truncated at the zero and first orders. Finally, as third case, he considered this factor as a constant in the calculation of the covariance matrix.
The same approximation were made by Chenavier and Crowley (Chenavier, 1992) and by Lu and Milios (Feng, 1995). They always considered a particular path and they used a Taylor approximation to compute the covariance matrix.

Finally Chong and Kleeman (Chong, 1997) divided the entire path in $N$ small segments. They found for the first time a closed form solution for the covariance matrix $Q$ as $N$ approaches infinity. In this way they did not require to do the Taylor approximation. However with their method they were able to compute this matrix only for special cases. Their expressions were applicable to only circular arc motion with constant radius of curvature.

In this paper we derive general formulas for the covariance matrix. In these formulas, applicable to any path, the trajectory of the robot motion appears as a function of the curve length. In these formulas there are some parameters which depend on the robot and on the environment where the robot moves.

The theoretical hypothesis about the odometry error are given in Section 2. In Section 3 we evaluate the covariance matrix and we show its dependence on the odometry error model parameters and on the robot motion. Finally the results are discussed in Section 4 together with some possible future investigation.

\section{THE ODOMETRY ERROR MODEL}

Assuming a two-dimensional world, we can define the robot configuration with respect to a worldcoordinate frame $W$ by the vector $X=[x, y, \theta]^{T}$, containing its position and orientation. The robot configuration estimated by odometry measurements is different from the actual configuration $X$ because of the odometry errors.

Consider the motion in the figure 1 . The curve represents the real motion followed by the robot. Let $x(\rho), y(\rho)$ and $\theta(\rho)$ be respectively the position and the orientation of the robot parameterized with respect to the curve length $\rho$ (the reference is chosen in such a way that at $\rho=0$ corresponds the origin). Given two points $A$ and $B$ we have $\widehat{\delta} \rho=\rho_{B}-\rho_{A}$ and $\widehat{\delta} \theta=\theta\left(\rho_{B}\right)-\theta\left(\rho_{A}\right)$.

We assume that both systematic and non-systematic errors only depend on $\widehat{\delta} \rho$ and do not depend on $\widehat{\delta} \theta$. This approximation can be done only for synchronous drive system and the more the trajectory is smooth the more the approximation holds.

Because of both systematic and non-systematic errors the encoder measurements $\overline{\delta \rho}$ and $\overline{\delta \theta}$ differ from the real values

$$
\widehat{\delta} \rho=\overline{\delta \rho}+E_{T} \overline{\delta \rho}+\delta \rho
$$




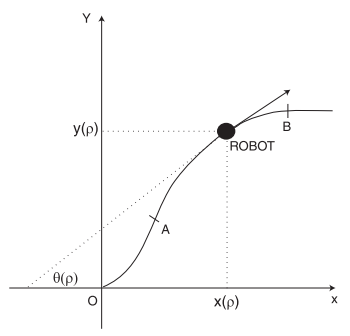

Fig. 1. The robot motion.

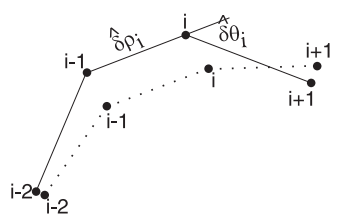

Fig. 2. The robot motion is divided in $N$ segments. The robot is assumed to move straight in each given segment. The solid line represents the real motion followed by the robot when $N \rightarrow \infty$, while the dotted line is the motion trajectory measured by the encoder

$$
\widehat{\delta} \theta=\overline{\delta \theta}+E_{R} \overline{\delta \rho}+\delta \theta
$$

where $E_{T} \overline{\delta \rho}$ and $E_{R} \overline{\delta \rho}$ represent the systematic components and $\delta \rho$ and $\delta \theta$ the non-systematic components.

Actually the systematic component is proportional to the real distance traveled by the robot (see for example (Roy, 1999)), i.e., instead of the equations (1) and (2) we should write:

$$
\begin{aligned}
& \widehat{\delta} \rho=\overline{\delta \rho}+E_{T}^{\prime} \widehat{\delta} \rho+\delta^{\prime} \rho \\
& \widehat{\delta} \theta=\overline{\delta \theta}+E_{R}^{\prime} \widehat{\delta} \rho+\delta^{\prime} \theta
\end{aligned}
$$

Equation (3) coincides with equation (1) once we set $1+E_{T}=\frac{1}{1-E_{T}^{\prime}}$ and $\frac{\delta^{\prime} \rho}{1-E_{T}^{\prime}}=\delta \rho$. On the other hand the equation (4) is different from (2). In fact by setting $E_{R}^{\prime}=\frac{E_{R}}{1+E_{T}}$ there is still the term $\frac{E_{R}}{1+E_{T}} \delta \rho$ which depends on $\delta \rho$. However this term is negligible and therefore from now on we use the equations (1) and (2).

Let consider a general robot motion. We approximate the trajectory with $N$ small segments. In figure 2 the solid line represents the real motion followed by the robot when $N \rightarrow \infty$. The dotted line is the motion trajectory measured by the encoder.

We introduce the following assumptions about the actual motion:

- the robot moves straight along each given segment whose length, measured by the encoder, is always the same value $\overline{\delta \rho}=\frac{\bar{\rho}}{N}$;

- the angle $\widehat{\delta} \theta_{i}$ between the orientations related to the $(i+1)^{t h}$ and the $i^{t h}$ segment and the translation $\widehat{\delta} \rho_{i}$ covered during the same step are gaussian random variables;

- the random variable $\widehat{\delta} \rho_{i}$ is independent of the random variable $\widehat{\delta} \theta_{i}$. Moreover $\widehat{\delta} \rho_{i}$ is independent of $\widehat{\delta} \rho_{j}(i \neq j)$ and $\widehat{\delta} \theta_{i}$ is independent of $\widehat{\delta} \theta_{j}$.

Let consider the $i^{t h}$ segment. From the previous assumptions we can write:

$$
\begin{aligned}
& \widehat{\delta} \rho_{i} \sim N\left(\overline{\delta \rho}\left(1+E_{T}\right), \sigma_{\delta \rho}^{2}\right) \\
& \widehat{\delta} \theta_{i} \sim N\left(\overline{\delta \theta_{i}}+E_{R} \overline{\delta \rho}, \sigma_{\delta \theta}^{2}\right)
\end{aligned}
$$

where $\overline{\delta \theta_{i}}$ is the angle between the orientations related to the $(i+1)^{t h}$ and the $i^{t h}$ segment measured by encoder and $\sigma_{\delta \theta}^{2}$ and $\sigma_{\delta \rho}^{2}$ are directly related to the rolling conditions.

The actual orientation after the $i^{t h}$ step is:

$$
\widehat{\theta_{i}}=\theta_{0}+\overline{\Delta \theta_{i}}+i E_{R} \overline{\delta \rho}+\sum_{j=1}^{i} \delta \theta_{j}
$$

where $\theta_{0}$ is the initial orientation of the robot and $\overline{\Delta \theta_{i}}$ is the global change in orientation measured by encoder.

By defining

$$
\widetilde{\theta}_{i}=\theta_{0}+\overline{\Delta \theta_{i}}+i E_{R} \overline{\delta \rho}
$$

and

$$
\Delta \theta_{i}=\sum_{j=1}^{i} \delta \theta_{j}
$$

we obtain

$$
\widehat{\theta_{i}}=\widetilde{\theta}_{i}+\Delta \theta_{i}
$$

where $\Delta \theta_{i}$ is still a random variable satisfying the following relation (Papoulis, 1991)

$$
\Delta \theta_{i} \sim N\left(0, i \sigma_{\delta \theta}^{2}\right)
$$

So far we have introduced the parameters $E_{R}$ and $E_{T}$ characterizing the systematic components of the odometry errors. We introduce now two new parameters $\left(K_{\theta}\right.$ and $\left.K_{\rho}\right)$ in order to characterize the non-systematic components, namely the two variances $\sigma_{\delta \theta}^{2}$ and $\sigma_{\delta \rho}^{2}$.

From the definition of $\overline{\delta \rho}=\frac{\bar{\rho}}{N}$ we can write:

$$
\sigma_{\theta}^{2}=N \sigma_{\delta \theta}^{2}=\bar{\rho} \frac{\sigma_{\delta \theta}^{2}}{\overline{\delta \rho}}
$$

namely there is a linear dependence of the variance $\sigma_{\theta}^{2}$ on the distance $\bar{\rho}$ measured by encoder. We therefore introduce the parameter $K_{\theta}$, whose 
estimation strategy is discussed in the section 4, defined by the following relation

$$
K_{\theta}=\lim _{N \rightarrow \infty} \frac{\sigma_{\delta \theta}^{2}}{\overline{\delta \rho}}
$$

We therefore have

$$
\sigma_{\theta}^{2}=K_{\theta} \bar{\rho}
$$

In the same way we compute the variance $\sigma_{\rho}^{2}$ obtaining:

$$
\sigma_{\rho}^{2}=K_{\rho} \bar{\rho}
$$

where

$$
K_{\rho}=\lim _{N \rightarrow \infty} \frac{\sigma_{\delta \rho}^{2}}{\overline{\delta \rho}}
$$

\section{THE COVARIANCE MATRIX Q}

The non-systematic errors are expressed in terms of the covariance matrix $\mathrm{Q}$. The robot configuration $X$ is a random vector whose average value $\langle X\rangle$ is given by the odometry measurements (once both the systematic errors are known). The covariance matrix $\mathrm{Q}$ is defined as follows:

$$
\begin{array}{r}
Q=E\left\{[X-<X>][X-<X>]^{T}\right\}= \\
=\left[\begin{array}{ccc}
\sigma_{x}^{2} & \sigma_{x y} & \sigma_{x \theta} \\
\sigma_{x y} & \sigma_{y}^{2} & \sigma_{y \theta} \\
\sigma_{x \theta} & \sigma_{y \theta} & \sigma_{\theta}^{2}
\end{array}\right]
\end{array}
$$

This matrix, of course, depends on the path followed by the robot and also on the type of floor surface. It can be represented as a function of the previous parameters $\left(E_{R}, E_{T}, K_{\theta}\right.$ and $\left.K_{\rho}\right)$, which can be determined experimentally as suggested in the following section.

\section{$3.1 \sigma_{\theta}^{2}$}

This matrix entry is expressed by the equation (14)

\section{$3.2 \sigma_{x}^{2}$}

We have

$$
\sigma_{x}^{2}=<x^{2}>-<x>^{2}
$$

In order to compute this matrix entry we need to express the final position coordinate $x$ in terms of $\widehat{\delta} \rho_{i}$ and $\widehat{\theta_{i}}$. We have:

$$
x=\lim _{N \rightarrow \infty} x_{N}=\lim _{N \rightarrow \infty} \sum_{i=1}^{N} \widehat{\delta} \rho_{i} \cos \left(\widehat{\theta}_{i}\right)
$$

Let begin to compute $\langle x\rangle$. In order to do this we compute firstly $\left\langle x_{N}\right\rangle$ and then we take the limit value when $N \rightarrow \infty$. We have:

$$
\begin{gathered}
<x_{N}>=\int d \widehat{\delta} \rho_{1} \ldots d \widehat{\delta} \rho_{N} d \widehat{\delta} \theta_{1} \ldots d \widehat{\delta} \theta_{N} f_{G}\left(\widehat{\delta} \rho_{1}, \sigma_{\delta \rho}\right) \ldots \\
\ldots f_{G}\left(\widehat{\delta} \rho_{N}, \sigma_{\delta \rho}\right) f_{G}\left(\widehat{\delta} \theta_{1}, \sigma_{\delta \theta}\right) \ldots f_{G}\left(\widehat{\delta} \theta_{N}, \sigma_{\delta \theta}\right)\{ \\
\left.\sum_{i=1}^{N} \widehat{\delta} \rho_{i} \cos \left(\widehat{\theta_{i}}\right)\right\}
\end{gathered}
$$

where we denoted with $f_{G}\left(w, \sigma_{w}\right)$ the gaussian distribution function of the random variable $w$ whose variance is $\sigma_{w}$.

By a direct calculation we obtain:

$$
<x_{N}>=\overline{\delta \rho}\left(1+E_{T}\right) \sum_{i=1}^{N} \cos \left(\widetilde{\theta}_{i}\right) e^{-\frac{i \sigma_{\delta \theta}^{2}}{2}}
$$

When $N \rightarrow \infty$ the sum in equation (21) becomes an integral. We obtain

$$
<x>=\lim _{N \rightarrow \infty}<x_{N}>=\left(1+E_{T}\right) \int_{0}^{\bar{\rho}} \cos (\tilde{\theta}(s)) e^{-\frac{K_{\theta} s}{2}} d s(22)
$$

where $\widetilde{\theta}(s)$ is the robot orientation as measured by encoder (included the systematic component) as a function of the curve length $s$ always measured by encoder which does not include the systematic component.

A little bit more troublesome is the computation of the second order product average $<x^{2}>$. By a direct calculation we obtain:

$$
\begin{gathered}
<x^{2}>=\left(1+E_{T}\right)^{2} \int_{0}^{\bar{\rho}} d s \int_{0}^{\bar{\rho}-s} d s^{\prime}\left\{e^{-\frac{K_{\theta} s^{\prime}}{2}} \times\right. \\
\times\left[\left(1+\chi_{C}(s)\right) \cos \left[\tilde{\theta}\left(s+s^{\prime}\right)-\tilde{\theta}(s)\right]+\right. \\
\left.\left.-\chi_{S}(s) \sin \left[\widetilde{\theta}\left(s+s^{\prime}\right)-\widetilde{\theta}(s)\right]\right]\right\}+ \\
+\frac{K_{\rho}}{2}\left[\bar{\rho}+\int_{0}^{\bar{\rho}} \chi_{C}(s) d s\right]
\end{gathered}
$$

where we denote with $\chi_{C}(s)=\cos [2 \widetilde{\theta}(s)] e^{-2 K_{\theta} s}$ and with $\chi_{S}(s)=\sin [2 \widetilde{\theta}(s)] e^{-2 K_{\theta} s}$.

$3.3 \sigma_{y}^{2}$

We have:

$$
\sigma_{y}^{2}=<y^{2}>-<y>^{2}
$$


The position coordinate $y$ in terms of $\widehat{\delta} \rho_{i}$ and $\widehat{\theta_{i}}$ is now:

$$
y=\lim _{N \rightarrow \infty} y_{N}=\lim _{N \rightarrow \infty} \sum_{i=1}^{N} \widehat{\delta} \rho_{i} \sin \left(\widehat{\theta_{i}}\right)
$$

Following the same procedure as in the previous case we obtain:

$$
<y>=\left(1+E_{T}\right) \int_{0}^{\bar{\rho}} \sin (\widetilde{\theta}(s)) e^{-\frac{K_{\theta} s}{2}} d s
$$

and

$$
\begin{gathered}
<y^{2}>=\left(1+E_{T}\right)^{2} \int_{0}^{\bar{\rho}} d s \int_{0}^{\bar{\rho}-s} d s^{\prime}\left\{e^{-\frac{K_{\theta} s^{\prime}}{2}} \times\right. \\
\times\left[\left(1-\chi_{C}(s)\right) \cos \left[\widetilde{\theta}\left(s+s^{\prime}\right)-\widetilde{\theta}(s)\right]+\right. \\
\left.\left.+\chi_{S}(s) \sin \left[\widetilde{\theta}\left(s+s^{\prime}\right)-\widetilde{\theta}(s)\right]\right]\right\}+ \\
+\frac{K_{\rho}}{2}\left[\bar{\rho}-\int_{0}^{\bar{\rho}} \chi_{C}(s) d s\right]
\end{gathered}
$$

$3.4 \sigma_{x y}$

We have:

$$
\sigma_{x y}=<x y>-<x><y>
$$

Therefore we only need to compute the second order product average $\langle x y\rangle$. We obtain:

$$
\begin{gathered}
<x y>=\left(1+E_{T}\right)^{2} \int_{0}^{\bar{\rho}} d s \int_{0}^{\bar{\rho}-s} d s^{\prime}\left\{e^{-\frac{K_{\theta} s^{\prime}}{2} \times}\right. \\
\times\left[\chi_{S}(s) \cos \left[\widetilde{\theta}\left(s+s^{\prime}\right)-\widetilde{\theta}(s)\right]+\right. \\
\left.\left.+\chi_{C}(s) \sin \left[\widetilde{\theta}\left(s+s^{\prime}\right)-\widetilde{\theta}(s)\right]\right]\right\}+ \\
+\frac{K_{\rho}}{2} \int_{0}^{\bar{\rho}} \chi_{S}(s) d s
\end{gathered}
$$

\section{$3.5 \sigma_{x \theta}$}

From the equation (10) we have for the final orientation $\widehat{\theta}$

$$
\widehat{\theta}=\lim _{N \rightarrow \infty} \widehat{\theta}_{N}=\lim _{N \rightarrow \infty}\left(\widetilde{\theta}_{N}+\Delta \theta_{N}\right)=\widetilde{\theta}+\Delta \theta(30)
$$

where we defined $\Delta \theta=\lim _{N \rightarrow \infty} \Delta \theta_{N}$
We have

$$
\sigma_{x \theta}=\langle x \Delta \theta>
$$

By a direct calculation we obtain:

$$
\sigma_{x \theta}=-\left(1+E_{T}\right) \int_{0}^{\bar{\rho}} \sin (\widetilde{\theta}(s)) K_{\theta} s e^{-\frac{K_{\theta}}{2} s} d s
$$

$3.6 \sigma_{y \theta}$

Following the same procedure as in the previous case we obtain:

$$
\sigma_{y \theta}=\left(1+E_{T}\right) \int_{0}^{\bar{\rho}} \cos (\widetilde{\theta}(s)) K_{\theta} s e^{-\frac{K_{\theta}}{2} s} d s(33
$$

Previous equations enable us to compute the elements of the matrix Q once the parameters $E_{R}$, $E_{T}, K_{\theta}$ and $K_{\rho}$ are known and the trajectory $\widetilde{\theta}(s)$ is specified.

\section{DISCUSSION}

In this paper we presented a theory about the odometry error for a mobile robot with a synchronous drive system with particular attention on its non-systematic component. We derived closed-formulas for the odometry error covariance matrix applicable, for the first time, to any path. In these formulas there are four parameters $\left(E_{R}\right.$, $E_{T}, K_{\theta}$ and $K_{\rho}$ ) which depend on the robot and on the environment where the robot moves.

We are developing a possible strategy to experimentally estimate the error parameters by only requiring to measure the change in the orientation and in the position between the initial and the final configuration of the robot related to suitable robot motions. In other words this strategy does not require to know the actual path followed by the robot. Moreover we are investigating on the possibility to apply this strategy to the platform Nomad150 in the most difficult case of an indoor environment, i.e. where the non-systematic components of the odometry error are very small and very difficult to be evaluated.

\section{REFERENCES}

Anousaki and Kyriakopoulos (1999). Simultaneous localization and map building for mobile robot navigation. IEEE Transactions on Robotics pp. $42-53$. 
Borenstein (1994a). Internal correction of deadreckoning errors with the smart encoder trailer. In: IROS '94, International Conference on Intelligent Robots and Systems. Vol. 1. pp. 127-134.

Borenstein (1995a). The clapper: A dual-drive mobile robot with internal correction of deadreckoning errors. In: ICRA '98, International Conference on Robotics and Automation. Vol. 3. pp. 3085-3090.

Borenstein (1998). Experimental results from internal odometry error correction with the omnimate mobile robot. IEEE Transactions on Robotics and Automation 14, 963-969.

Borenstein, Feng (1994b). Umbmark - a method for measuring, comparing and correcting dead-reckoning errors in mobile robots. In: Technical Report UM-MEAM-94-22, University of Michigan.

Borenstein, Feng (1995b). Correction of systematic odometry errors in mobile robots. In: IROS '95, International Conference on Intelligent Robots and Systems. Vol. 3. pp. 569574.

Borenstein, Feng (1996). Measurement and correction of systematic odometry errors in mobile robots. IEEE Transactions on Robotics and Automation 12, 869-880.

Chenavier, Crowley (1992). Position estimation for a mobile robot using vision and odometry. In: ICRA '92, International Conference on Robotics and Automation. Vol. 3. pp. 25882593.

Chong, Kleeman (1997). Accurate odometry and error modelling for a mobile robot. In: ICRA '97, International Conference on Robotics and Automation. Vol. 4. pp. 2783-2788.

Feng, Milios (1995). Optimal global pose estimation for consistent sensor data registration. In: ICRA '95, International Conference on Robotics and Automation. Vol. 1. pp. 93-100.

Goel, Roumeliotis, Sukhatme (1999). Robust localization using relative and absolute position estimates. In: IROS '94, International Conference on Intelligent Robots and Systems. Vol. 2. pp. 1134-1140.

Papoulis, A. (1991). Probability, Random Variables, and Stochastic Process. McGRAWHILL INTERNATIONAL EDITIONS.

Roy, Thrun (1999). Online self-calibration for mobile robots. In: ICRA '99, International Conference on Robotics and Automation. Vol. 3. pp. 2292-2297.

Wang (1988). Location estimation and uncertainty analysis for mobile robots. In: ICRA '88, International Conference on Robotics and Automation. pp. 1231-1235.

Zhejun, Borenstein, Wehe Koren (1995). Experimental evaluation of an encoder trailer for dead-reckoning in tracked mobile robots. In:
IEEE International Symposium on Intelligent Control. pp. 571-576. 